\title{
Valuation of Pumped Storage in Capacity Expansion Planning-A South African Case Study
}

\author{
Caroline van Dongen, Bernard Bekker (1) and Amaris Dalton * \\ Department of Electrical and Electronic Engineering, Stellenbosch University, Stellenbosch 7600, South Africa; \\ caroline.vandongen@arup.com (C.v.D.); bbekker@sun.ac.za (B.B.) \\ * Correspondence: amaris_dalton@sun.ac.za
}

Citation: van Dongen, C.; Bekker, B.; Dalton, A. Valuation of Pumped Storage in Capacity Expansion Planning-A South African Case Study. Energies 2021, 14, 6999. https://doi.org/10.3390/en14216999

Academic Editors: Pavlos S. Georgilakis and Andrea Mariscotti

Received: 30 July 2021

Accepted: 4 October 2021

Published: 26 October 2021

Publisher's Note: MDPI stays neutral with regard to jurisdictional claims in published maps and institutional affiliations.

Copyright: (c) 2021 by the authors. Licensee MDPI, Basel, Switzerland. This article is an open access article distributed under the terms and conditions of the Creative Commons Attribution (CC BY) license (https:// creativecommons.org/licenses/by/ $4.0 /)$.

\begin{abstract}
According to South Africa's national energy policy, network penetration of variable renewable energy (VRE) generation will significantly increase by 2030. Increased associated network uncertainty creates the need for an additional flexible generation. As the planned VRE is mostly nonsynchronous PV and wind generators, additional ancillary services will also be required. Pumped Storage (PS), which is a well-established flexible generation technology with fast ramping capability and the ability to contribute various ancillary services, could help integrate increased VRE penetration on the South African network. However, in the latest revision of South Africa's energy policy, PS was left out in favor of gas turbines and batteries as favored flexible generation options. This paper explores the two-part hypothesis that PS was disadvantaged in the formulation of a national energy mix due to: (a) ancillary services provided by PS not being explicitly monetized in energy modeling software; (b) the uncertainties associated with project costing assumptions. The value of PS in terms of providing ancillary services is firstly explored using the international literature. Secondly, the impact of input-cost uncertainties is demonstrated by comparing pumped storage, gas turbines, and batteries using levelized cost of energy (LCOE) curves and the Tools for Energy Model Optimization and Analysis (Temoa), North Carolina State University, USA, optimization software. Based on LCOE calculations using revised cost assumptions, it is found that PS may indeed be preferential to gas turbines or batteries, particularly at large load factors. The authors hope that this research contributes to the scientific understanding of the role that PS can play in supporting the integration of generation from renewable sources for effective grid operations.
\end{abstract}

Keywords: pumped storage; ancillary services; variable renewable energy; gas turbine; flexible generation

\section{Introduction}

Variable renewable energy (VRE), notably wind and solar photovoltaic (PV), is rapidly proliferating onto electricity networks around the world. Indeed, in the latest revision of South Africa's key energy policy document, the integrated resource plan (IRP), published in October 2019, the South African government envisions an ambitious increase in VRE capacity from a baseline of approximately $6 \%$ in 2020 to $26 \%$ by 2030 [1]. To offset the generation uncertainty associated with VRE, the need for grid-scale and flexible electricity storage solutions is becoming critically important. Mature storage technologies include pumped storage (PS), electrochemical batteries, flywheels, and compressed-air energy storage. Of these technologies, PS is the most mature and widely utilized utility-scale technology [2], comprising approximately $96 \%(1310 \mathrm{GW})$ of installed storage capacity as of 2019 [3]. For a more detailed discussion on the international, and specifically the South African development of PS, the reader referred to [4] where it is noted that PS has played a fairly limited role in South Africa's electricity mix in large part due to the country's arid climate. Notwithstanding South Africa's climate, it is furthermore argued in [4] that the country has sufficient water resources and adequate locations for PS to play an important role in South Africa's future energy mix. However, despite the international dominance of 
PS as storage technology and the sufficiency of South Africa's resources, additional PS was not included in the IRP 2019 as an intended part of South Africa's future energy mix [1].

Long-term energy planning is critical in informing a country's energy mix to ensure that future energy demand is met at minimum cost while supporting various policy objectives such as commitments to decarbonization. In South Africa, the national power utility, Eskom, along with the Department of Energy (DoE), utilize energy modeling to identify the most compatible and cost-effective energy alternatives to meet the country's future energy demands. As such, modeling was key to the formulation of the IRP. By modeling different scenarios, alternative energy mixes can be compared whereby the costs and benefits for each scenario are estimated. Two processes are typically used for long-term energy modeling. Firstly, capacity expansion modeling, which models a few selected days in a typical year for the next, e.g., thirty years, and optimizes the energy mix based on certain criteria. Secondly, adequacy assessment modeling is used to simulate a full year at a much higher resolution for a selected scenario.

This paper explores the two-part hypothesis that PS may be disadvantaged in the formulation of a national energy mix due to: (a) certain ancillary services provided by PS not being explicitly monetized in energy modeling software; (b) the large uncertainties that might exist in project costing assumptions that serve as inputs into energy modeling software. The study hypothesis is informed by a literature review, which highlights that methodologies and model input values for representing storage during capacity expansion modeling are not yet well defined, especially in the interaction between variable renewable energy generators and storage technologies. Issues such as chronology, capacity value, and cost representation have yet to be addressed adequately in most large-scale modeling frameworks used for this purpose.

The proposed hypothesis was tested by using the exclusion of PS from the IRP 2019 as a case study. The above hypothesis was specifically investigated from two perspectives in this paper. Firstly, in Section 2, the cost of PS and its value specifically in relation to the provision of ancillary services are explored at the hand of international literature. Within this context, the South African case is then considered, analyzing the constraint inherent in the fact that current modeling software used to inform the IRP is not capable of accounting for the value of ancillary services separately. In Sections 3 and 4, the second perspective is explored, i.e., the sensitivity of the modeling outcomes to uncertainty in the technology costings used as inputs in capacity expansion modeling. Section 3 compares PS, gas, and batteries through levelized cost of energy (LCOE) calculations where PS costs used to inform earlier revisions of the IRP are compared to those used in later revisions of the IRP, which were solely informed by a single (approximately $400 \%$ over-budget and four years delayed) South African pumped storage project called Ingula. Section 4 builds on Section 3 by exploring the impact of various PS cost inputs using the open-source Temoa optimization energy modeling software. Section 5 concludes the paper and offers recommendations. Figure 1 provides an outline of the paper.
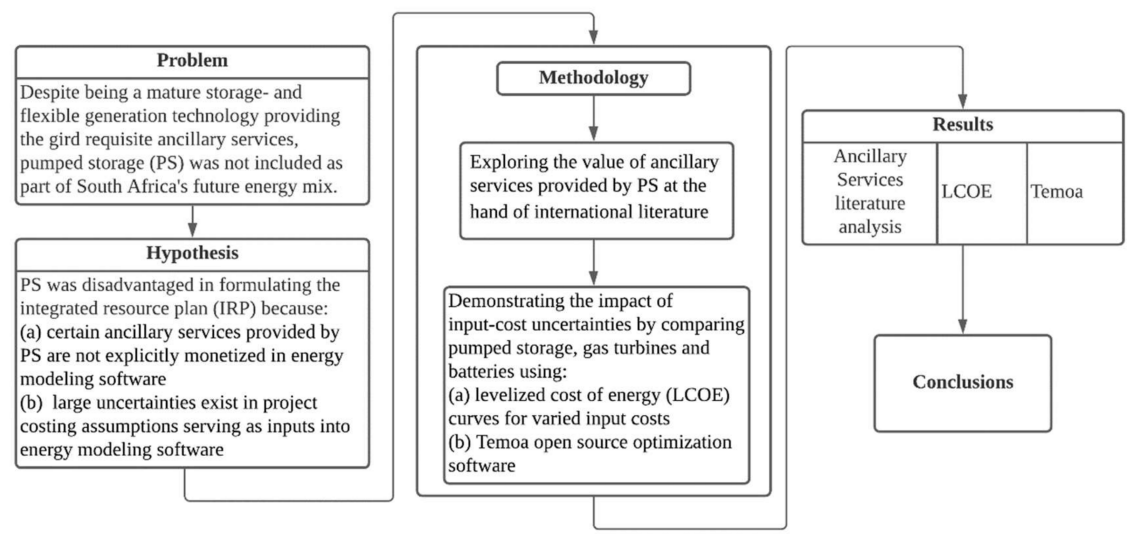

Figure 1. Study Outline. 


\section{Valuation of PS Ancillary Services in Capacity Expansion Planning \\ 2.1. Value and Cost of PS Ancillary Services Internationally}

As an energy storage technology, PS supports a wide variety of power system operations through various ancillary services. These services range from inertia response and flexible ramping to primary/secondary frequency control and reduced curtailment of VRE [5]. The financial value of such services is expected to increase as the percentage of VRE sources in the power system increases. Indeed, a recent study estimated that ancillary services and energy arbitrage in the US offered by $100 \mathrm{MW}$ of storage could result in yearly revenues in excess of approximately R435 million (USD 30 million (note for continuity, all currencies values in this paper were converted to South African Rand $(R)$ at the approximate 2021 exchange rate of R14.5 to the USD)) [6]. This includes frequency regulation up/down at R141/MWh and for Australia ancillary services (lower and raise, $6 \mathrm{~s}, 5 \mathrm{~min}$, regulation, restart, and reactive) at $\mathrm{R} 153 / \mathrm{MW}$ [6].

The lack of explicit ancillary service monetization may stem from difficulties in evaluating the monetary value services such as inertia response, voltage support, transmission deferral, and energy security [5]. In the US, for example, PS can only receive revenue for limited services, including electricity generation, regulation reserve, spinning reserve, nonspinning reserve, and the provision of black-start capability arranged through a long-term contract. A recent study conducted by the United States Department of Energy in collaboration with the National Renewable Energy Laboratory (NREL), Siemens and Energy Exemplar, specifically focused on the modeling and analysis of the value of PS [5]. The value of PS was determined by calculating power system production cost savings and revenue analyses using Energy Exemplar's PLEXOS Integrated Energy Model model, North Adelaide, Australia. The benefits and value of PS were analyzed for different types of PS in both regulated and competitive electricity market environments in the United States. The study highlighted that in both traditional and restructured energy markets, ancillary services and contributions that PS provides to the power grid are not fully monetized [7]. It was calculated that PS should receive R145/MW for up/downregulation, R72.5/MW for increase/decrease flexibility, and R14.5-43.5/MW for spinning and non-spinning reserves [5].

As with the monetary value of ancillary services provided by PS, international estimated costs of PS technologies differ widely. Costs are dependent both on the geographic location of the PS installation and the type of PS unit used. PS units generally fall within three categories of technology: fixed speed, variable/adjustable speed, and ternary. Compared to the traditional fixed-speed units, adjustable speed units can adjust the rate at which water is pumped, thereby giving more regulation services, while ternary units have a separate pump and turbine, which allows for higher flexibility, efficiency, and response times as quick as $25 \mathrm{~s}$ [8]. The estimated costs for adjustable speed units are 10-20 percent higher than for fixed speed units [9]. For a $10 \mathrm{~h}, 300$ to $1000 \mathrm{MW}$ plant, 2017 costs were estimated to be in the wide range of R24650-R73950/ kW. Estimates from the US Department of Energy in 2019 have placed PS between R24650/kW and R46 400/kW, averaging $\mathrm{R} 38251 / \mathrm{kW}$ [10]. These costs are, however, estimates based on various assumptions and dependent on a particular market structure; therefore, it is important to understand PS within a South African context and the ancillary services it provides.

\subsection{Value and Function of PS on the South African Grid}

This section provides an overview of the range of ancillary services that PS provides and could provide to the South African grid. Eskom defines ancillary services as reserve frequency support, islanding, reactive power supply, voltage control, constrained generation, and black starts [11], and PS is instrumental in the provision of each of these.

Firstly, with regards to frequency response, Figure 2 illustrates the activation and sustained times of reserve for Eskom in response to frequency deviations outside the dead band of $49.85 \mathrm{~Hz}$ to $50.15 \mathrm{~Hz}$ [11]. Eskom's required peak operating reserves for 2020/21 are $650 \mathrm{MW}$ instantaneous (primary) reserves, $500 \mathrm{MW}$ regulating (secondary) reserves, 
and $1050 \mathrm{MW}$ ten-min (tertiary) reserves [12]. From an emergency point of view, PS can be used both as a black start facility and also arrest a frequency drop following a severe frequency event, such as a multi-contingency trip. Hydro generators have the advantage of stable operations during a Rate of Change of Frequency (RoCoF) events ranging from $0.5 \mathrm{~Hz} / \mathrm{s}$ to $2 \mathrm{~Hz} / \mathrm{s}$ [13].

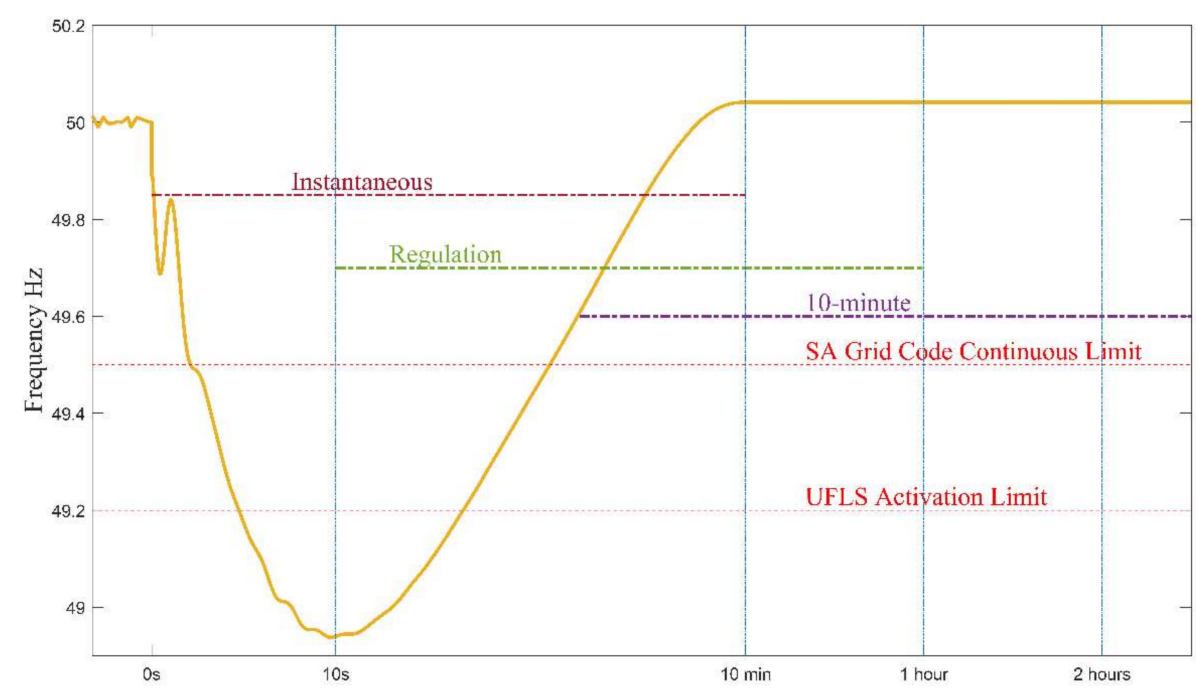

Figure 2. Activation and sustained times of reserve for Eskom to restore frequency during a significant system fault. Partially adopted from $[14,15]$.

On the South African grid, the PS units' response to frequency deviations is controlled by the primary governor, which is installed at the local plant level and is activated when the grid frequency is not within the established dead band limits. The System Operator ensures that there is always PS capacity in reserve, except during the morning and evening peak times as pumped storage is used to compensate for the slower ramp rates of Open Cycle Gas Turbines (OCGTs) and coal-fired generators [11]. This improves the economics of the overall system, as excessive ramping reduces operating efficiency for thermal plants. Indeed, thermal plants are typically designed for high constant output, whereby excessive ramping leading to more equipment stress and increased maintenance costs. Over the nightly minimum load demand period, water is typically pumped back to the upper reservoir, which adds significant demand to the system allowing baseload generators to remain synchronized to the power system, thereby increasing the baseload utilization level and optimizing operating costs.

Coal units on automatic generation control (AGC) are used for the regulation reserve and operate at partial load to increase their output to balance the minute-by-minute supply and demand. PS units in South Africa are currently fixed speed units rather than on AGC and thereby operate in the 10-min reserve. PS in the reserve allows the system operator to export 200-250 MW to the grid in approximately 2-3 min, whereby the PC response speed significantly exceeds that of a coal station, which is only $15 \mathrm{MW} / \mathrm{min}$ [14].

PS is not just used for reserves and for black starts but also to provide reactive power and voltage control services [11]. The need for voltage control may also become increasingly important of future energy networks as increased distributed VRE may negatively impact centralized voltage regulation, which is typically achieved using an on-load tap changer, because significant reverses in power flow may cause the substation busbar not to be the point of greatest voltage magnitude [16]. PS units are synchronous machines and, when unloaded, can act as synchronous condensers. The PS generator supplies reactive power to the grid, which can also function as a capacitor bank. In large sizes, synchronous condensers are cheaper than capacitor banks and provide convenient and continuous control of reactive power by adjusting the field current. Generally, there are one or two hy- 
dro/PS units at a plant generating or providing voltage control through Synchronous Condenser Operation (SCO) mode [14]. This mode adds inertia and voltage control capability to the network. The addition of network inertia arrests the RoCoF and may increase the network frequency nadir during contingency events. Similar to voltage control, it is anticipated that the sufficiency of network inertia will become increasingly topical on the South African electricity network owing to the planned decommissioning of five large synchronous coal-fired generators coupled with substantial increases in VRE by 2030 [1]. Indeed, inertia challenges have already been observed in a number of networks owing to increased VRE [17-19]. Voltage control, in turn, decreases fault levels in the network and smooths out variation in voltage caused by load changes and disturbances. Presently, the PS units provide around -3 MW output during SCO pump mode [14], whereby changes in mode are estimated at 5 to 20 times a day per unit by Eskom Operators [20].

Currently, fixed speed turbines used in South Africa have a non-minimum phase response, which means that immediately after a request for a power increase, which opens the guide vanes, the power momentarily drops before increasing to the new setpoint. This means frequency regulation using fixed-speed pumped storage is not recommended [14]. However, internationally, PS was installed with variable speed turbines, which can also be used in frequency regulation [21]. Further design improvements were also developed with different arrangements where, for example, the turbine runner may be placed on its own runner shaft with a flywheel between turbine and generator which decreases hydraulic shock (water hammer) and can increase the unit's requirements for synchronization, isolated mode, and grid conditions.

\subsection{Analysis}

As was demonstrated in the previous sections, PS provides a range of ancillary services to the power system, whereby many of these services are set to become increasingly topical owing to the increased proliferation of VRE onto the network, along with the simultaneous planned decommissioning of synchronous generators. Market-valued monetization of such services has, however, proven a continual challenge where a range of prices are observed, depending on the market structure and grid characteristics of the specific region.

Specific to South Africa, PS operations currently fall within a vertically integrated national utility where its services are not explicitly valued [20]. This is about to change, with Eskom being unbundled, resulting in an independent system operator and the IRP allocating more than $26 \%$ of the country's total generating capacity to VRE by 2030 . Within this context, an ancillary services market will likely develop, resulting in a more realistic valuation of PS-supplied ancillary services. Current capacity expansion modeling in South Africa is performed using an analytic tool called PLEXOS, developed by Energy Exemplar. At the time of modeling the IRP, the model used did not explicitly monetize ancillary services.

In summary, it is argued that the monetization of ancillary services and the employment of appropriate cost-input assumptions are crucial in the establishment of a scrupulous national energy policy. This is demonstrated through an analysis of the pricing inputs informing the IRP 2019, which it is argued, should be reconsidered based on the IRP 2019 's failure to consider the monetary value of ancillary services provided by various storage technologies during the optimization modeling. Practically, we propose that this shortcoming is expressed as a costing bias against PS.

\subsection{Recommendations}

International markets with day-ahead dispatch as a rule value the different ancillary services and consider marginal energy costs, start-up costs, ramping rates, operating reserves, and transmission constraints. The current ancillary services provided by PS in South Africa can be valued by analyzing such international market mechanisms, especially concerning the spinning/non-spinning, flexible ramping up and down, and reactive power/voltage control. Potential revenue from frequency regulation can also be included 
for future variable turbines with improvements in turbine generator arrangements. In system planning and economic operation of power systems, generators are typically not operated at their limits but at equal incremental costs (taking into consideration the line losses) on an instantaneous basis. PS, however, is different as the incremental costs are not known. The cost varies as it may be based on excess VRE or generation from thermal power stations [20]. Therefore, prediction and forecasting are required to allow for PS to reduce the overall production cost of the system by generating at the peak times, lowering capacity building, storing excess energy generation, and operating during off-peak to allow for constant operation of the thermal units at night [22].

\section{PS Costs as Inputs into Capacity Expansion Modelling}

\subsection{PS Allocations in Recent South African IRPS}

Consecutive revisions of the IRP throughout the previous decade have seen a significant increase in the assumed costs of PS. Indeed, previous costing assumptions modeled PS at R7 913/kW in 2010 IRP [23], which steeply increased to R21 997/kW in the draft 2018 IRP modeling [24] upon which the finalized 2019 IRP is based. Current local estimates for costs of PS in South Africa ranges from R13 000/kW [25] to R22 000/kW [26] based on the recently constructed Ingula PS and the feasibility study for the proposed Kobong PS scheme. It is reasonable to hypothesize that, as a result of these significantly increased costs, PS has not been included in new capacity solutions for South Africa in the IRP 2019. This may have allowed for other technologies such as OCGT and batteries [27] to replace PS as the main generating capacity for peaking generation in South Africa's future energy mix, as shown in IRP 2019 [1].

Section 3 explores the second part of the stated study hypothesis that the IRP's failure to include PS is based on inaccurate costing assumptions used during the capacity expansion modeling process-primarily informed by the high Ingula PS project costs.

\subsection{LCOE Technology Inputs and Assumptions}

The main PS input assumptions in the PLEXOS model used in the establishment of the IRP were compared against the primary, flexible generation competing technologies, i.e., OCGT and lithium-ion batteries. As a cost optimizing tool, PLEXOS chooses the lowest cost scenario using a mixed-integer programming algorithm for mathematical optimization.

In order to test the PLEXOS results, the cost inputs used in the IRP for each technology were used to create Levelized Cost of Energy (LCOE) curves whereby a power plant should be designed to have the lowest LCOE over the lifetime of the plant to ensure the viability of the project. The LCOE represents the mean required revenue per unit generated, which is required for the projected capital, operating, and fuel expenses associated with an assumed generator's life cycle to be recovered [28]. The LCOE is thereby the mean net present cost of electricity produced throughout a generator's life cycle. LCOE is perhaps the most widely used tool to comparatively different benchmark technologies across a lifetime of energy supply costs $[29,30]$. Though LCOE provides an aggregate figure, it is flexible and can be used as a cost assessment tool that can incorporate technical factors (for example, ramping rate, system costs, and construction time), economic factors (for example, financing, interest rates, costs related to geographic location and fuel prices) and even externalities such as environmental impacts [29]. LCOE was thereby utilized to compare the competing technologies as it measures lifetime costs divided by energy production [31].

LCOE for a power plant is calculated by the following formula [31]:

$$
L C O E=\frac{\text { Sum of discounted costs over lifetime }}{\text { Sum of electric energy over the lifetime }}=\frac{\sum_{t=1}^{n} \frac{C_{t}+O M_{t}}{(1+r)^{t}}}{\sum_{t=1}^{n} \frac{E_{t}}{(1+r)^{t}}}
$$

where $C_{t}=$ the capital cost expenditure in a year; $O M_{t}=$ the Operation and Maintenance $(\mathrm{O} \& \mathrm{M})$ expenditure in a year; $E_{t}=$ the annual energy generation $(\mathrm{kWh}) ; \mathrm{r}=$ the economic discount rate, and $n=$ the lifetime of the plant. 
By calculating and comparing the LCOEs of PS and the competing flexible generation technologies, value can be measured across the longer term, showing projected life-cycle costs. Each of the technologies was calculated for the different load factors for each plant. The load factor, also known as the capacity factor, refers to the ratio between the actual power output of a generator over a period and the power it would have produced if it had been operating at its rated capacity for the same period.

\subsection{LCOE Technology Inputs and Assumptions}

The LCOEs for PS, OCGT, and lithium-ion batteries were calculated using the PLEXOS modeling inputs from the IRP 2010 [23], IRP 2016 [32], and draft IRP 2018 [24], wherein the modeling results was published on which the final IRP 2019 was based. In order to compare the sensitivity of the PLEXOS model to its initial inputs, the different costs for PS were included from the feasibility studies for Tubatse PS [33] and Kobong PS [25], and different fuel prices were used to compare OCGT costs. Tables 1 and 2 show the data used to develop the curves.

Table 1. Input parameters from IRP 2010 and 2016.

\begin{tabular}{|c|c|c|c|c|c|}
\hline Technology Input & $\begin{array}{l}\text { Open cycle gas turbine } \\
\text { (OCGT) Integrated } \\
\text { Resource Plan (IRP) } \\
2010 \text { Diesel R7.26/L }\end{array}$ & $\begin{array}{c}\text { Pumped } \\
\text { Storage(PS) IRP } \\
2010\end{array}$ & $\begin{array}{l}\text { OCGT IRP } 2016 \\
\text { Liquified Natural } \\
\text { Gas (LNG) } \\
\text { R116/GJ }\end{array}$ & $\begin{array}{l}\text { OCGT IRP } \\
2016 \text { Diesel }\end{array}$ & $\begin{array}{c}\text { PS IRP } 2016 \\
\text { R7.26/L }\end{array}$ \\
\hline Cost overnight $(\mathrm{R} / \mathrm{kW})$ & R3955 & R7913 & R7472 & R7472 & R20410 \\
\hline Fuel Cost $(\mathrm{R} / \mathrm{GJ})$ & $\mathrm{R} 200$ & - & $\mathrm{R} 115$ & $\mathrm{R} 200$ & 0 \\
\hline Capacity (MW) & 114.7 & 1500 & 132 & 132 & 333 \\
\hline O\&M Variable (R/MWh) & 0 & 4 & 2.2 & 2.2 & 0 \\
\hline O\&M Fixed $(\mathrm{R} / \mathrm{kW} / \mathrm{a})$ & R70 & R123 & R147 & $\mathrm{R} 147$ & R184 \\
\hline Lifetime of project (years) & 30 & 50 & 30 & 30 & 50 \\
\hline Discount rate $(\%)$ & 8 & 8 & 8.2 & 8.2 & 8.2 \\
\hline Phasing in Capital Spent (\%) & 90,10 & $\begin{array}{c}3,16,17,21,20 \\
14,7,2\end{array}$ & 90,10 & 90,10 & $\begin{array}{c}1,2,9,16,22, \\
24,20,5\end{array}$ \\
\hline
\end{tabular}

Table 2. Input parameters from IRP 2019 with Kobong and Tubatse PS.

\begin{tabular}{|c|c|c|c|c|c|c|c|}
\hline Technology Input & $\begin{array}{c}\text { OCGT } \\
\text { LNG } \\
\text { R66.2/GJ }\end{array}$ & $\begin{array}{l}\text { Ingula } \\
\text { PS }\end{array}$ & $\begin{array}{l}\text { Lithium-Ion } \\
\text { (1 h) }\end{array}$ & $\begin{array}{l}\text { Lithium-Ion } \\
\qquad(3 \mathrm{~h})\end{array}$ & Kobong PS & Tubatse PS & $\begin{array}{l}\text { OCGT } \\
\text { Diesel } \\
\text { R10.8/1 }\end{array}$ \\
\hline Cost overnight $(\mathrm{R} / \mathrm{kW})$ & R9226 & R21997 & R11165 & $\mathrm{R} 27432$ & R13389 & R16446 & R9226 \\
\hline Capacity (MW) & 132 & 333 & 3 & 3 & 1200 & 1500 & 132 \\
\hline O\&M Variable (R/MWh) & $\mathrm{R} 2.7$ & R0 & R3.6 & R3.6 & R0 & R0 & $\mathrm{R} 2.7$ \\
\hline O\&M Fixed (R/kW/a) & R181 & R184 & R697 & R697 & R184 & R184 & R181 \\
\hline Lifetime of project (years) & 30 & 50 & 20 & 20 & 50 & 50 & 30 \\
\hline Discount rate $(\%)$ & 8.2 & 8.2 & 8.2 & 8.2 & 8.2 & 8.2 & 8.2 \\
\hline Phasing in Capital Spent (\%) & 90,10 & $\begin{array}{c}1,2,9,16,22 \\
24,20,5\end{array}$ & 100 & 100 & $\begin{array}{c}14,8,12 \\
17,15,14,16\end{array}$ & $\begin{array}{c}1,2,9,16,22 \\
24,20,5\end{array}$ & 90,10 \\
\hline
\end{tabular}

The following assumptions were made to generate the curves:

- For the debt model, a total loan facility of the overnight cost amount was made available at rates of 8\% (IRP 2010) and 8.2\% (IRP 2016, 2019) for a period of 20 years. An assumed interest and capital payment moratorium were imposed for the first years of the loan, i.e., during the construction phase, then repaid as an amortized loan;

- $\quad$ For the IRP 2010, the pump storage fuel cost was calculated according to the EGEAS methodology [34], where the pumping cost for a pumped storage plant was based on the variable cost of coal of the "available" baseload plants in the system, i.e., baseload plants with a relatively high variable coal cost [33]. For purposes of comparing the LCOE curves, an average coal cost of R200/t was assumed. The variable component, 
i.e., the pumping cost, was based on a coal cost estimated at R200/MWh, as the energy charge (fuel) component of the total levelized cost;

- The draft IRP 2019 input values for OCGT and lithium-ion batteries were taken from the Electric Power Research Institute (EPRI) report 2018 [35], while the pumped storage costs were based on the Ingula PS scheme. The storage technologies do not include a marginal or variable cost for the power [24];

- Kobong and Tubatse feasibility costs used compounded South African inflation rate to give an estimate of the overnight costs.

It should finally be noted that, though not included in the results below, additional assessments focused on other cost factors such as fuel and O\&M were conducted. However, it was found that capital and fuel costs had the biggest impact on the LCOE analysis; these cost inputs, therefore, remained the focus of our analysis.

\subsection{LCOE Curves Results}

The results for the developed LCOE curves comparing the historic 2010 and 2016 IRPs based on PS and OCGT costs are shown in Figure 3. It is evident that at load factors of below 7\%, OCGT are the favored technologies after which they surpassed by PS based on the IRP 2010 costing assumptions, which includes a pumping "fuel" cost. When comparing the competitiveness of PS based on the IRP 2010 vs. the IRP 2016 costing assumptions, Figure 3 thereby clearly illustrates how the increase in PS costing assumptions based on Ingula's costs at R20 410/kW [32] rendered PS significantly less competitive, especially at lower load factors. Therefore, PS was replaced by OCGT LNG at R116/GJ as the new competing technology in IRP 2016.

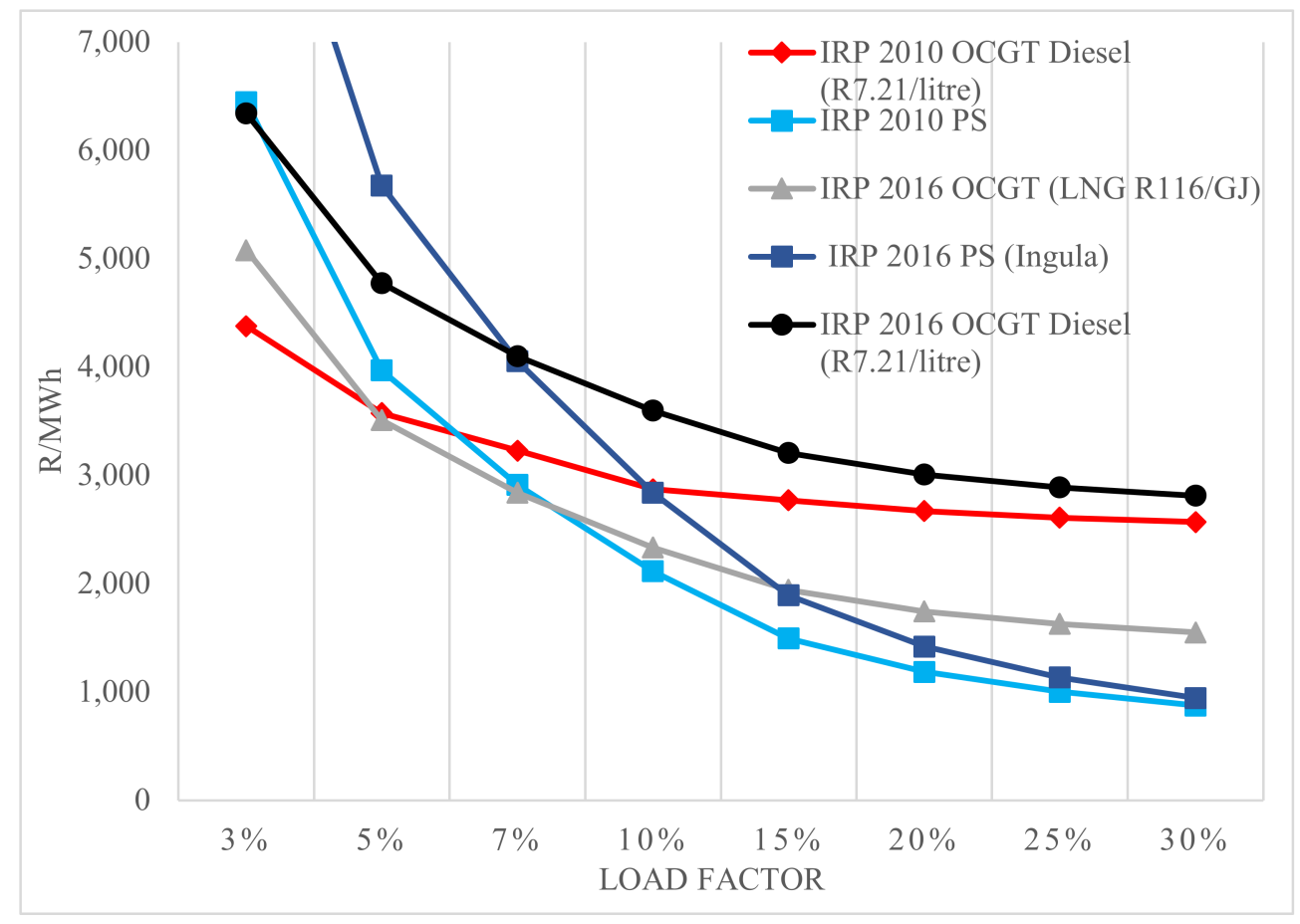

Figure 3. LCOE curves for IRP 2010 and 2016 comparing PS and OCGT.

Figure 4 shows LCOE curves based on the 2019 IRP where a number of OCGT LNG and diesel costing scenarios along with 1 and $3 \mathrm{~h}$ battery storage is compared against PS as informed by Ingula's costing assumptions. It is demonstrated how, under these conditions, OCGT has replaced PS as the favored technology, especially at low load factors. Diesel peaking plants and lithium-ion batteries are generally shown to be the least costeffective solutions. 


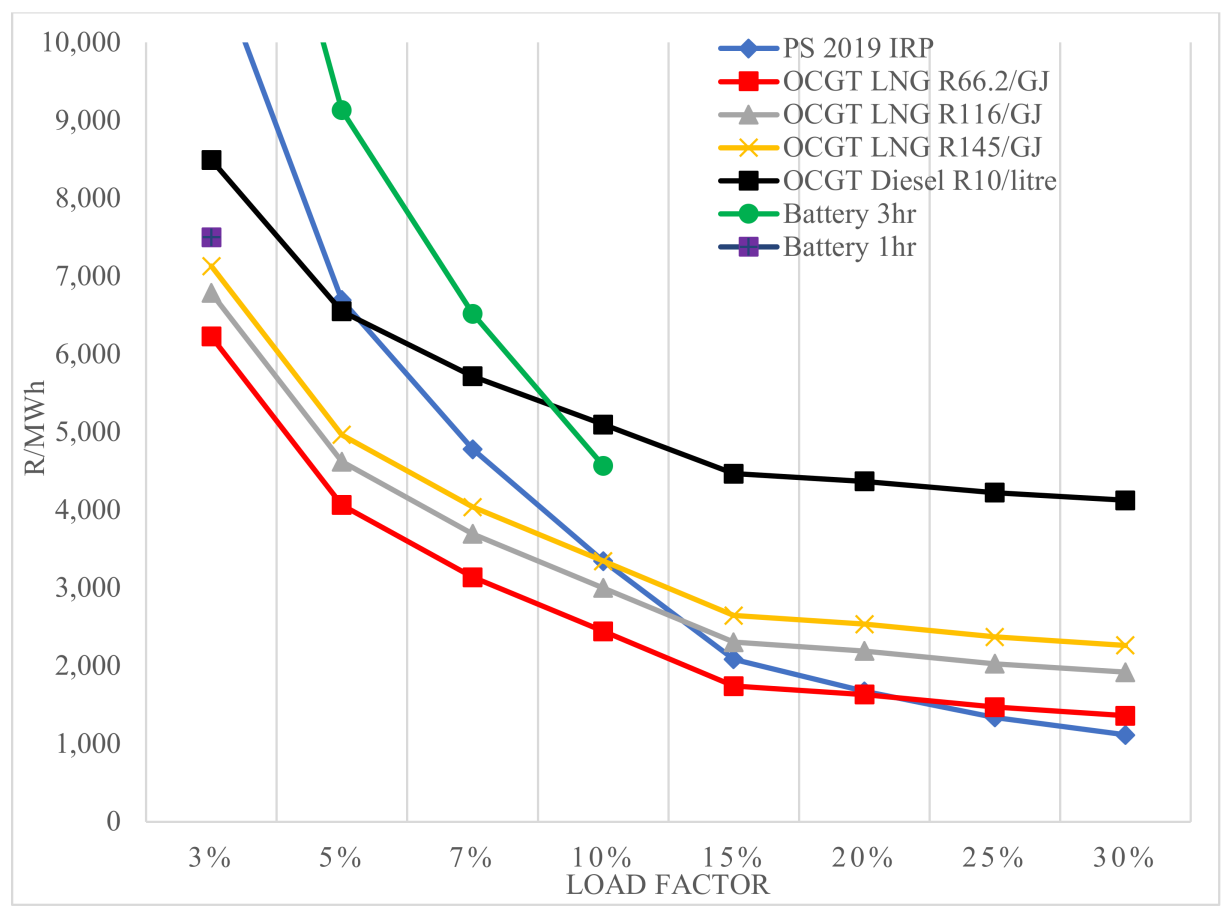

Figure 4. LCOE curves for IRP 2019 comparing PS (informed by Ingula), OCGT, and lithiumion battery.

For further emphasis on the impact the using of Ingula's costs as model input parameters had, Figure 5 provides a comparison for the IRP 2019 PS (with Ingula) against alternative PS costing inputs based on assessments from the planned Kobong and Tubatse PS schemes. It is evident that the preferred scheme is Kobong PS, which competes well with the lowest OCGT LNG costing assumption (the best performing option in Figure 4) at load factors of greater than $5 \%$.

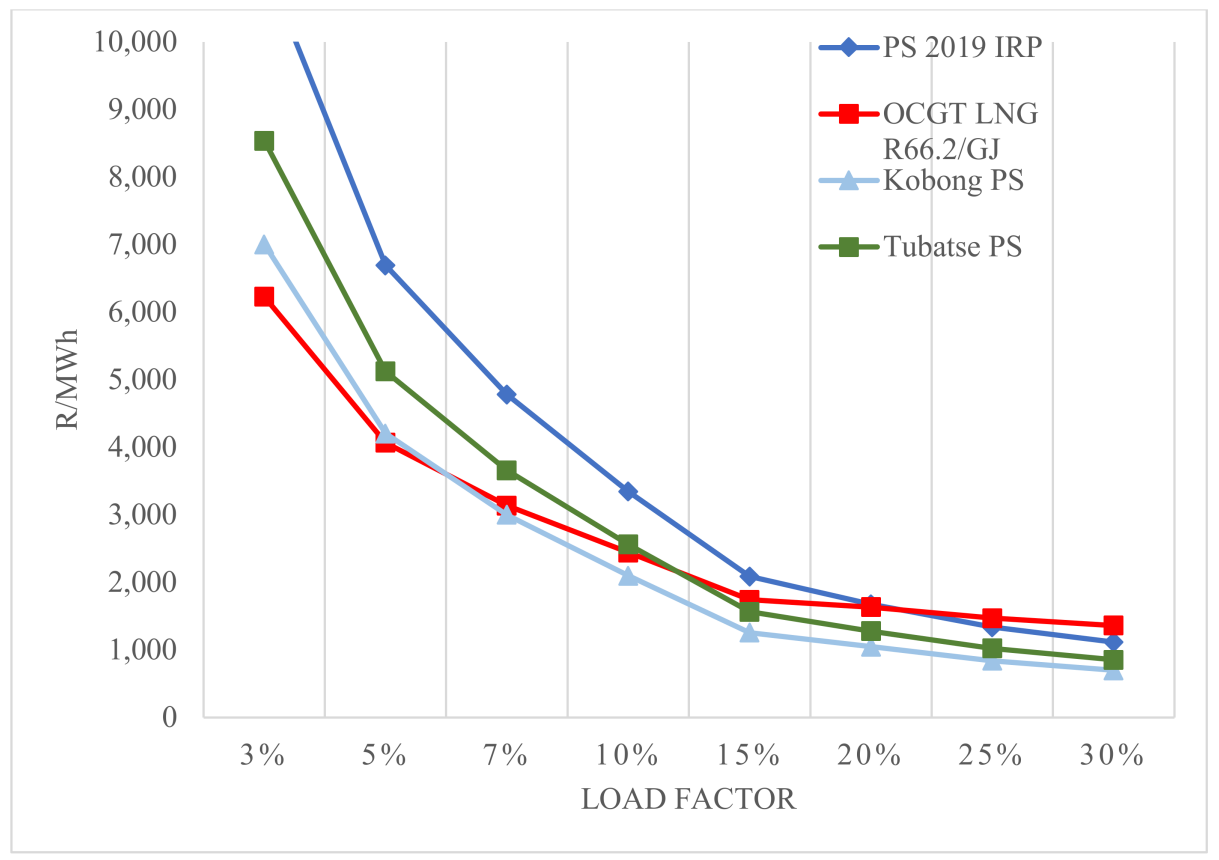

Figure 5. LCOE curves for IRP 2019, including alternative costing inputs based on Tubatse and Kobong. 


\subsection{Analysis}

The levelized costs for each technology were given for certain load factors in the IRP reports. These values were used to compare and validate the developed LCOE curves. Figures $3-5$ show that, even without ancillary services being explicitly costed in, PS can be competitive with OCGT, especially with decreased capital costs. Therefore, an argument can be made for PS as a more cost-effective option for peaking generation than OCGT, especially at higher load factors.

These results also correspond to the outcomes of a study conducted in the US with capital costs for PS at R30 000/kW and gas turbines at R9 400/kW [5], which shows that lowered capital costs for PS, with ancillary services cost in, would be competitive with gas turbines. A similar study conducted in the US [10] in 2019 found PS to be more cost-effective than lithium-ion and OCGT, even when valued at R37 000/kW.

\section{Energy Model Optimization and Analysis}

In order to gain a holistic understanding of how the differences in chosen cost inputs of PS affected the system model, the paper hypothesis had to be tested in an energy model which could replicate the South African grid. As the authors did not have access to the original PLEXOS model, Tools for Energy Model Optimization and Analysis (Temoa) was used [36]. Temoa is an open-source energy supply costs optimization framework for modeling power systems implemented in Python using the Pyomo package.

Temoa was designed to address difficulties associated with uncertainty analysis in complex system models. To this effect, Temoa enables an assessment of several economic and technical properties associated with a range of generation technologies and how they interact within a power system. Generation technologies are defined in terms of several economic, engineering, and environmental parameters. In terms of its functionality, Temoa defines a power system numerically as a network of connected processes that convert an unprocessed product (e.g., coal, oil, uranium) into an end-use service (e.g., lighting, heating, transport) through an intermediate service (e.g., electricity, gasoline).

The inputs used to create the model were based on the IRP 2019 forecast, EPRI, and the Council for Scientific and Industrial Research (CSIR)'s formal comments on the IRP 2016 [37]. In order to simplify the model, wind and solar were considered as renewables, and nuclear power and hydropower were grouped together. As part of model verification, results were checked against the IRP 2019 results for 2030, 2040, and 2050.

\section{Temoa Modelling Results}

It was found that the Temoa modeling results correlated well with the IRP 2019, 2030, 2040, and 2050 capacity results. However, the IRP2019 selected gas turbine capacity growth from $6.3 \mathrm{GW}$ to $23,24 \mathrm{GW}$ from 2030 to 2050, while Temoa chose rather to include $24 \mathrm{GW}$ OCGT from 2030. The same resultant capacity was still, however, chosen, so this minor deviation was considered acceptable. Another point to note is that the IRP 2019 is not clear on future storage requirements after 2030. Therefore, the model was checked to match the 2030 requirements and left to estimate the system requirements for 2040 and 2050 within the same energy mix as IRP 2019.

The initial run was performed as a validation exercise where similar inputs were used for the IRP 2019. Results are shown in Figure 6, which are comparable to the IRP 2019 proposed energy mix. Subsequently, the PS costing inputs were altered to gain an understanding of what the impact of these changes would be on system model output results. The capital cost was changed from the initial modeled value of R30 450/kW to Kobong's R13862/kW. From Figure 7, it is evident that gas turbine capacity requirement dropped by almost half from $24 \mathrm{GW}$ to $12,7 \mathrm{GW}$ leading to 2050, while the PS capacity requirement doubled for 2030 to $10 \mathrm{GW}$ and increased from 10,9 GW to 23,9 GW by 2050 . 


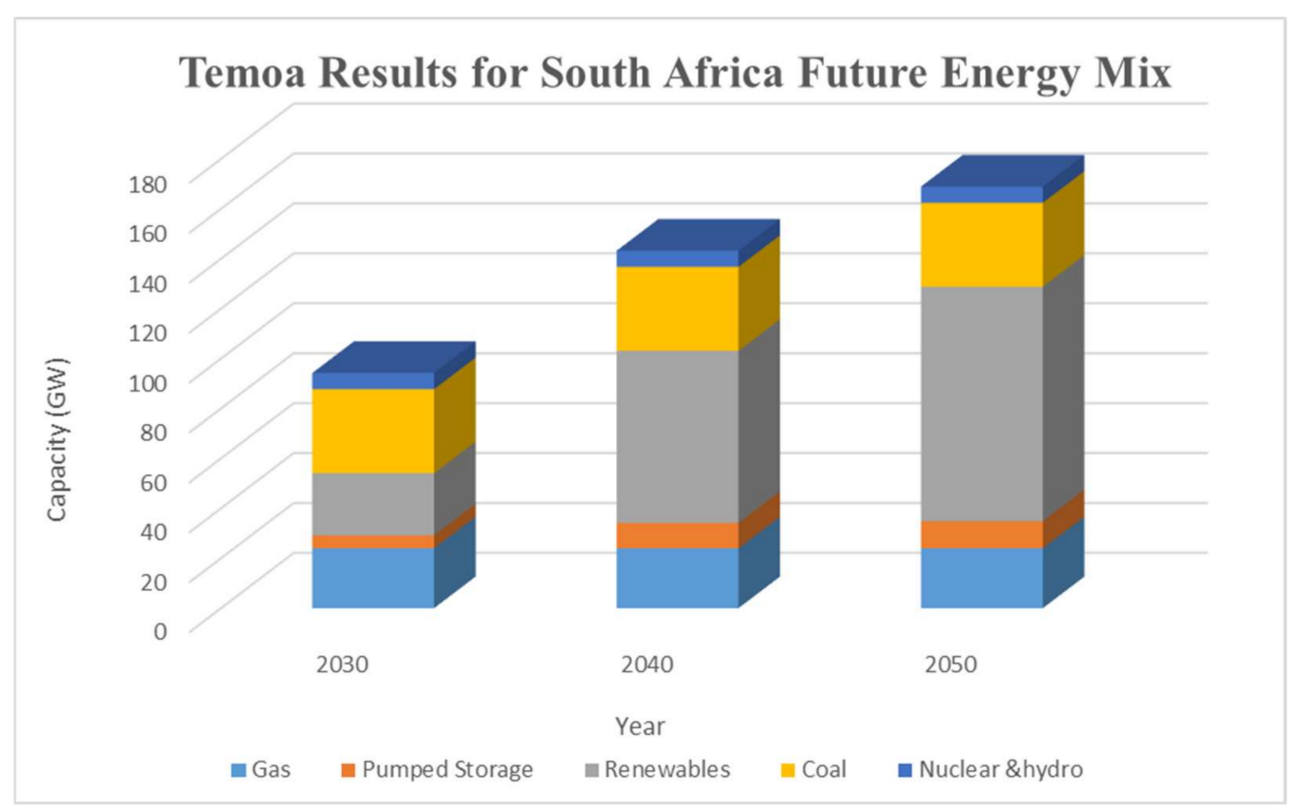

Figure 6. Temoa Results for South Africa Future Energy Mix using the same inputs as the IRP 2019 for PS.

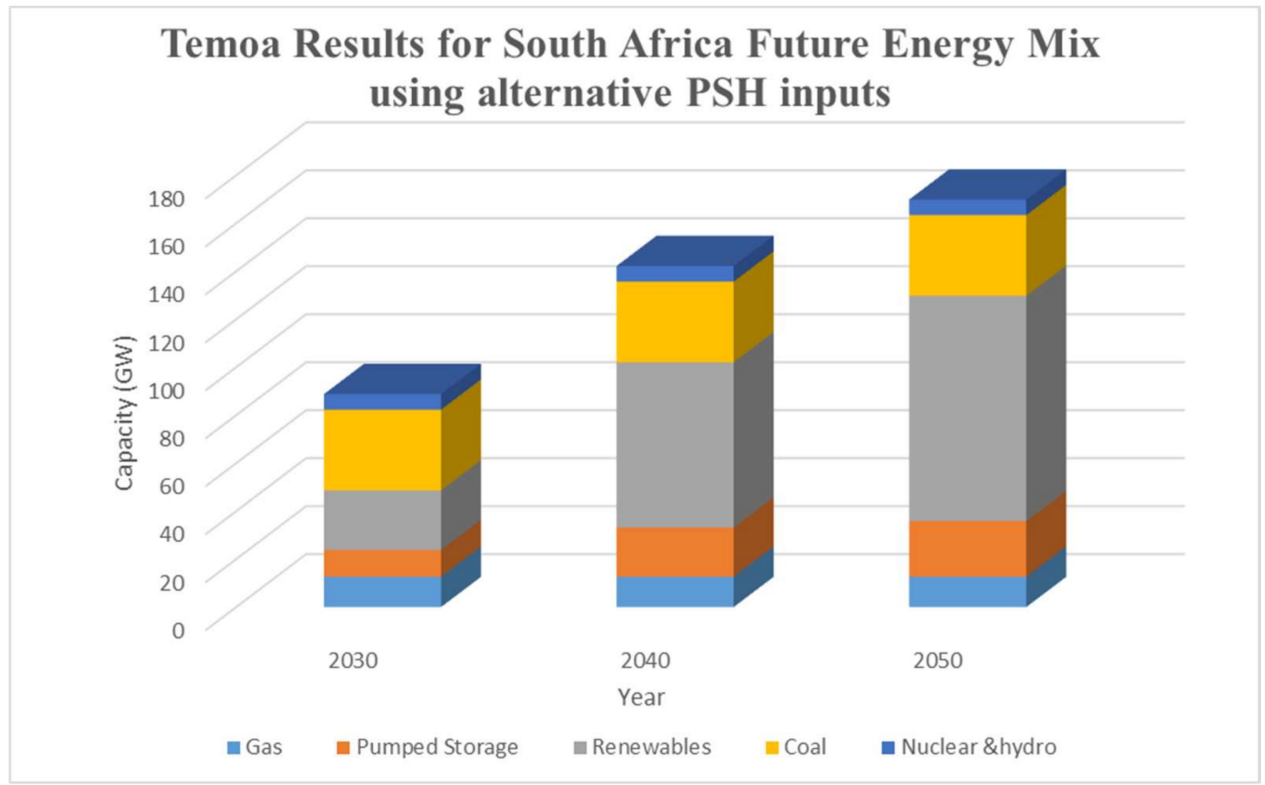

Figure 7. Temoa Results for South Africa Future Energy Mix using alternative PS inputs.

The Temoa model is thereby deemed to provide a good indication of the potential changes in PS development potential if different PS costs were to be used as PLEXOS model inputs.

\section{Conclusions}

This paper explored the hypothesis that PS may be disadvantaged in the formulation of a national energy mix when the ancillary services it provides are not monetized and based on costing inputs used during the optimization modeling. The hypothesis is tested using a case study centered around the exclusion of PS from the most recent South African IRP.

The paper focused on the costs related to pumped storage and the ancillary services the technology provides the grid. It showed that, on a power system dominated by baseload coal-fired generation, PS offers flexibility, ramping speed, inertia support, voltage control, 
and demand during low loading periods. Currently, these services are not monetized for PS on the South African grid due to the grid's vertically integrated nature. Future studies should include additional revenue for these services in the energy model to understand the benefits that PS provides to the grid.

The input assumptions for PS in the PLEXOS model for the IRP were investigated further through the development of LCOE curves as an alternative costing methodology. The LCOE curves supported the hypothesis that an economic case can be made for PS in South Africa, depending on how PS is modeled in the energy planning software. The results showed PS to have significantly lower LCOE values than batteries and diesel turbines, particularly at higher load factors. The cost competitiveness of PS and gas turbines was shown to be dependent on the gas price used and the chosen PS overnight cost.

Finally, the impact of incorporating new modeling inputs for PS into the optimization model was explored using the Temoa software. When using cost estimates from the Kobong PS, rather than the significantly overbudget and delayed Ingula project, the optimum PS capacity requirement increases significantly from current IRP 2019 estimates.

The LCOE curves and Temoa model assisted in supporting the hypothesis that an economic case can still be made for PS in South Africa. This is, however, dependent on how PS is currently modeled in the energy planning software. It is thereby concluded that the chosen input costs of PS should be derived from a representative composite based on a number of projects and feasibility studies rather than on a single previous project.

In terms of this study's limitations and avenues for future work, it should be noted that there are various uncertainties associated with input cost assumptions and net future value calculations that require further investigation. Notable examples of such uncertainties include: future OCGT fuel costs, particularly within the context of decreasing gas prices but also considering infrastructure requirements related to LNG; the rand/dollar exchange rate, which is particularly pertinent in potentially higher inflationary environments as may be anticipated in an emerging market; uncertainties relating to the validity and transparency of discount rates used in LCOE analysis; and, as discussed in detail in Section 2, uncertainties relating to the value of ancillary services.

Finally, the authors hope that this research may contribute to the scientific understanding of the potential role that PS can play in improving the integration of generation from renewable sources for effective grid operations. This is highly topical, especially in a coal-dominated electricity system, such as South Africa's, to create an opportunity for a sustainable transition to a more environmentally conscious energy future. In highlighting certain potential pitfalls that may be avoided in the formulation of a national energy policy, it is further anticipated that this paper would be beneficial to network planners and policy experts perusing a renewables-orientated energy transition.

Author Contributions: Conceptualization, C.v.D.; methodology, C.v.D.; software, C.v.D.; validation, C.v.D. and B.B.; formal analysis, C.v.D.; investigation, C.v.D.; resources, C.v.D.and B.B; data curation, C.v.D.; writing — original draft preparation, A.D.; writing—review and editing, A.D., B.B. and C.v.D.; visualization, C.v.D. and A.D.; supervision, B.B.. All authors have read and agreed to the published version of the manuscript.

Funding: This research received no external funding.

Institutional Review Board Statement: Not applicable.

Informed Consent Statement: Not applicable.

Data Availability Statement: Not applicable.

Acknowledgments: The authors would like to acknowledge the Eskom Power Plant Engineering Institute and the Centre for Renewable and Sustainable Energy Studies at Stellenbosch University, South Africa, for the funding and administrative support they provided.

Conflicts of Interest: The authors declare no conflict of interest. 


\section{References}

1. Department of Mineral Resources and Energy. Integrated Resource Plan 2019; Government Printing Works: Pretoria, South Africa, 2019; p. 100.

2. Gür, T.M. Review of electrical energy storage technologies, materials and systems: Challenges and prospects for large-scale grid storage. Energy Environ. Sci. 2018, 11, 2696-2767. [CrossRef]

3. Blakers, A.; Stocks, M.; Lu, B.; Cheng, C. A review of pumped hydro energy storage. Prog. Energy 2021, 3, 022003. [CrossRef]

4. Van Dongen, C.; Bekker, B. Potential for new pumped storage schemes in South Africa. In Proceedings of the 6th IEEE International Energy Conference, ENERGYCon 2020, Gammarth, Tunisia, 28 September-1 October 2020; pp. 266-271. [CrossRef]

5. Argonne National Laboratory. Modeling and Analysis of Advanced Pumped Storage Hydropower in the United States. In Proceedings of the HydroVision 2014, Nashville, TN, USA, 22-25 July 2014; 2014.

6. $\quad$ Lazard. Lazard's Levelised Cost of Storage Analysis-Version 4.0; Lazard: Hamilton, Bermuda, 2018.

7. National Hydropower Association. Pumped Storage Report; NHA: Washington, DC, USA, 2018.

8. Department of Energy. Request for Comments: Draft Integrated Resource Plan 2018. Government Notice; U.S. Department of Energy: Washington, DC, USA, 2018.

9. Guo, T.; Koritav, V. Adjustable Speed Pumped Storage Hydro Generator PSH Evaluate by Plexos; Energy Exemplar LLC: Salt Lake City, UT, USA, 2016.

10. Mongird, K.; Viswanathan, V.V.; Balducci, P.J.; Alam, M.J.E.; Fotedar, V.; Koritarov, V.S.; Hadjerioua, B. Energy Storage Technology and Cost Characterization Report; U.S. Department of Energy: Washington, DC, USA, 2019.

11. Eskom Holdings. System Operator-Ancillary Services Technical Requirements for 2019/20-2023/24; Eskom: Johannesburg, South Africa, 2015.

12. Tshwagong, I.; Ntusi, M.; Smith, V. System Operator-Ancillary Services Technical Requirements for 2020/21-2024/25; Eskom: Johannesburg, South Africa, 2020.

13. Chown, G. Frequency Presentation; Stellenbosch University-Centre for Renewable and Sustainable Energy Studies: Stellenbosch, South Africa, 2019.

14. Kippie, M. Improvements to a Key Contributor of Frequency Control: The Co-Ordination of Guide Vane Operation in a Pumped Storage Plant. Master's Thesis, University of Cape Town, Cape Town, South Africa, 2014.

15. Roux, A.; Bekker, B.; Dalton, A. Synchronous Condensers as a Viable Inertia Support Mechanism on the Future South African Grid; 2021; paper submitted for review.

16. Tshivhase, N.; Hasan, A.L.I.N.; Shongwe, T. A Fault Level-Based System to Control Voltage and Enhance Power Factor Through an On-Load Tap Changer and Distributed Generators. IEEE Access 2021, 9, 34023-34039. [CrossRef]

17. Gu, H.; Yan, R.; Saha, T. Review of System Strength and Inertia Requirements for the National Electricity Market of Australia. CSEE J. Power Energy Syst. 2019, 5, 295-305. [CrossRef]

18. Johnson, S.C.; Papageorgiou, D.J.; Mallapragada, D.S.; Deetjen, T.A.; Rhodes, J.D.; Webber, M.E. Evaluating rotational inertia as a component of grid reliability with high penetrations of variable renewable energy. Energy 2014, 180, 258-271. [CrossRef]

19. Mararakanye, N.; Bekker, B. Renewable energy integration impacts within the context of generator type, penetration level and grid characteristics. Renew. Sustain. Energy Rev. 2019, 108, 441-451. [CrossRef]

20. Binneman, R.; Hurford, G. Email correspondence. 2018.

21. Cole, W.; Frew, B.; Mai, T.; Sun, Y.; Bistline, J.; Blanford, G.; Young, D.; Marcy, C.; Namovicz, C.; Edelman, R.; et al. Variable Renewable Energy in Long-Term Planning Models: A Multi-Model Perspective; National Renewable Energy Lab.(NREL): Golden, CO, USA, 2017.

22. Gonzalez-Salazar, M.A.; Kirsten, T.; Prchlik, L. Review of the operational flexibility and emissions of gas-and coal-fired power plants in a future with growing renewables. Renew. Sustain. Energy Rev. 2018, 82, 1497-1513. [CrossRef]

23. Department of Energy. Integrated Resource Plan for Electricity; Government Printing Works: Pretoria, South Africa, 2011.

24. Department of Energy. Request for Comments: Draft Integrated Resource Plan 2018. Government Notice; Department of Energy, Integrated Resource Plan for Electricity: Pretoria, South Africa, 2011.

25. SWECO. Technical/Economic Feasibility Study on Kobong Pumped Storage Scheme. Financial Evaluation Report; SWECO: Stockholm, Sweden, 2013.

26. Kahla, C. Eskom's Defective R29-Billion Power Plant Limits Electricity Output. The South African. 27 March 2019. Available online: https:/ / www.thesouthafrican.com/news/eskom-power-plant-defective-r29-billion/ (accessed on 23 July 2021).

27. Barbour, E.; Wilson, I.A.G.; Radcliffe, J.; Ding, Y.; Li, Y. A review of pumped hydro energy storage development in significant international electricity markets. Renew. Sustain. Energy Rev. 2016, 61, 421-432. [CrossRef]

28. Lai, C.S.; McCulloch, M.D. Levelized cost of electricity for solar photovoltaic and electrical energy storage. Appl. Energy 2017, 190, 191-203. [CrossRef]

29. Hansen, K. Decision-making based on energy costs: Comparing levelized cost of energy and energy system costs. Energy Strategy Rev. 2019, 24, 68-82. [CrossRef]

30. Yates, T.; Hibberd, B. Levelized cost of energy. Solar Pro Magazine, April/May 2012.

31. Andrews, J.; Jelley, N. Energy Science: Principles, Technologies, and Impacts; Oxford University Press: Oxford, UK, 2017.

32. Department of Energy. Integrated Resource Plan Update: Assumptions, Base Case Results and Observations (Revision 1). Technical Report; U.S. Department of Energy: Washington, DC, USA, 2016. 
33. Eskom Holdings. Project Lima Pumped Storage Scheme; Business Case Report; Eskom: Johannesburg, South Africa, 2008.

34. Electric Power Research Institute. Electric Generation Expansion Analysis System (EGEAS); EPRI: Palo Alto, CA, USA, 1982.

35. Electric Power Research Institute. Energy Storage Technology and Cost Assessment: Executive Summary; EPRI: Palo Alto, CA, USA, 2018.

36. Howells, M.; Rogner, H.; Strachan, N.; Heaps, C.; Huntington, H.; Kypreos, S.; Hughes, A.; Silveira, S.; DeCarolis, J.; Bazillian, M.; et al. OSeMOSYS: The open source energy modeling system: An introduction to its ethos, structure and development. Energy Policy 2011, 39, 5850-5870. [CrossRef]

37. Wright, J.G.; Calitz, J.R.; Ntuli, N.; Rampokanyo, M.J.; Kamera, P.; Fourie, R. Formal Comments on Integrated Resource Plan (IRP) 2018: Presentation; CSIR Energy Centre: Pretoria, South Africa, 2018. 